\title{
Psicología positiva: algunas aplicaciones a la educación
}

Dr. Luis Rodríguez de Los Ríos

\section{Resumen}

La psicología positiva, considerada como una nueva visión dentro de la psicología, surge con la expectativa de conocer las características, los procesos y las condiciones del funcionamiento óptimo de las personas, para promover una vida plena y significativa desde la perspectiva individual hasta la proyección social. Este artículo aporta un análisis teórico-práctico de los conceptos y aplicaciones relativos al enfoque de la psicología positiva, los cuales son abordados desde el campo de la educación, fomentando un proceso de enseñanza-aprendizaje desde y para la felicidad. Establecer un camino educativo desde y para la felicidad implicaría por parte de los educadores, un compromiso personal de conocerse a sí mismos, tener valores y perseguir metas. La actual importancia de la psicología positiva dentro de la educación lleva a la necesidad de trabajar este tema con el fin de darle la transcendencia que merece por los efectos beneficiosos que tiene tanto en los docentes como en los estudiantes dentro de las aulas. Por ello debemos conocer las herramientas que proporciona para poder aplicarlas en el aula. Mediante las propuestas educativas, la felicidad de los estudiantes puede convertirse en una meta a alcanzar en el día a día, haciendo del aprendizaje y de la etapa de escolarización un período donde, a la vez que disfrutamos, aprendemos a ser felices en el aquí y ahora, sin descuidar por ello la importancia del pasado y el futuro.

\section{Palabras clave}

Psicología Positiva, Educación, Emociones, Fortalezas, Calidad de vida.

\section{Abstract}

Positive psychology, considered as a new vision within psychology, arises with the expectation of knowing the characteristics, processes, and conditions of the optimal functioning of people. This is used to promote a full and meaningful life from the individual perspective to the social projection. This article provides a theoretical-practical analysis of the concepts and applications related to the 
positive psychology approach; which is addressed from the field of education by promoting a teaching-learning process from and to happiness. Establishing an educational path from and to happiness would imply on the part of the educators a personal commitment to know themselves, to have values, and to pursue goals. The current importance of positive psychology within education leads to the need to work on this topic in order to give it the importance it deserves and show the beneficial effects it has on both teachers and students in an educational environment. Therefore, we must know the tools that it provides to be able to apply them in the classroom. Through the educational proposals, the happiness of the students can become a goal to reach in the day to day, making the learning and the stage of schooling, a period, in which we enjoy and also learn to be happy in the here and now without neglecting the importance of the past and the future.

\section{Keywords}

Positive Psychology, Education, Emotions, Strengths, Quality of life. 


\section{Introducción}

Hace más de dos décadas el surgimiento de la Psicología Positiva conmovió el campo de la Psicología tradicional al aportar una mirada salugénica e incorporar las fortalezas y los recursos de las personas como tema de estudio. Este movimiento, que todavía está en sus inicios y que viene creciendo en todo el mundo, cuenta cada día con más estudios e investigaciones, desarrollos y aplicaciones en diversos campos.

Se han alzado voces que, retomando las ideas de la psicología humanista acerca de la necesidad del estudio de la "parte positiva" de la existencia humana han aportado un sólido soporte empírico y científico a esta parte descuidada de la psicología. El término psicología positiva fue desarrollado por Seligman, que, habiendo dedicado gran parte de su carrera al trastorno mental y al desarrollo de conceptos como la indefensión aprendida, ha dado un giro radical en su orientación, elaborando y promoviendo una concepción más positiva de la especie humana. (Seligman y Csikszentmihalyi, 2000).

La Psicología Positiva, definida como el estudio científico del funcionamiento psíquico óptimo, se propone estudiar e investigar temas que fueron dejados de lado por las corrientes mayoritarias dentro de la Psicología orientadas a reparar el daño, temas tales como el amor, la capacidad de perdonar, la espiritualidad, el humor, los valores, las emociones positivas y la sabiduría, entre muchos otros.

En los últimos años se han llevado a cabo no sólo investigaciones básicas sobre estos temas, sino también se van desarrollando intervenciones y aplicaciones surgidas a partir de los estudios de la Psicología Positiva, tanto en el campo de la psicoterapia, como en la educación y en las organizaciones.

La Psicología Positiva se basa en tres pilares: en primer lugar es el estudio de la emoción positiva; el estudio de los rasgos positivos, sobre todo las fortalezas y virtudes, pero también las "habilidades" como la inteligencia y la capacidad atlética; y el estudio de las instituciones positivas, como la democracia, las familias unidas y la libertad de información, que sustentan las virtudes y a su vez sostienen las emociones positivas. La psicología positiva tiene como objetivo mejorar la calidad de vida y prevenir la aparición de trastornos mentales y patologías.

La concepción tradicional focalizada en lo patológico se centra en corregir defectos y reparar aquello que ya se ha roto. Por el contrario, la psicología positiva insiste en la construcción de competencias y en la prevención. El objeto de este interés no es otro que aportar nuevos conocimientos acerca de la psique humana no sólo para ayudar a resolver los problemas de salud mental que adolecen a los individuos, sino también para alcanzar mejor calidad de vida y bienestar, todo ello sin apartarse nunca de la más rigurosa metodología científica propia de toda ciencia de la salud.

\section{Antecedentes e Historia de la Psicología Positiva}

El desarrollo teórico e investigativo que ha dominado a la psicología a lo largo del tiempo ha estado centrado en las emociones negativas, y en la debilidad humana en general, dando lugar 
a un marco disciplinar sesgado hacia lo patogénico, aspecto que ha incidido en que la psicología sea identificada como psicopatología o psicoterapia (Strumpfer, 2004; Vera, 2006). Este marcado énfasis en lo patológico estuvo influido por la Segunda Guerra Mundial, período en el que la psicología se consolidó como una disciplina dedicada a la curación y a la reparación de daños (Seligman y Christopher, 2000), bajo el modelo biomédico dominante en ese entonces. No obstante antes de la guerra, los objetivos de la psicología no solo estaban orientados hacia la curación de los trastornos mentales, sino que sus acciones debían contribuir a que la vida de las personas fuera más productiva y plena, lo que implicaba identificar y desarrollar el talento y la inteligencia de las personas. Tras la guerra y por diferentes circunstancias, estos últimos objetivos fueron rezagados y la psicología se dedicó exclusivamente al tratamiento del trastorno mental y a aliviar el sufrimiento humano (Seligman y Csikszen-tmihalyi, 2000). Producto de dicha aproximación, casi exclusiva en lo patológico, la psicología ha desarrollado algunos modelos de intervención eficaces y eficientes para muchos problemas psicológicos, en detrimento del avance en métodos y estrategias para alcanzar y optimizar los recursos y fortalezas de los individuos, aspectos de los que actualmente no se disponen conocimientos sólidos. (Vázquez, 2006).

Si bien el bienestar y la felicidad han constituido temas de reflexión desde la antigüedad, por parte, de los filósofos y literatos en todos los tiempos. Desde la perspectiva psicológica se ubican como antecedentes de la psicología positiva a los trabajos de Willian James (1902), los escritos de Watson sobre el cuidado psicológico de los infantes, y a finales de los años 30, con el trabajo de Terman y colaboradores sobre el talento de los estudiantes y su ambiente académico y los factores psicológicos relacionados con la felicidad (Seligman y Csikszentmihalyi, 2000); las investigaciones de Allport (1958), las obras de representantes de la psicología humanista como Rogers (1951), Maslow (1954), Jahoda (1958), Erickson (1963) y Ryff y Singer (1996), entre otros. Asimismo, según Castro Solano (2009, citado por Lupano y Castro, 2010) muchos psicólogos han venido haciendo psicología positiva sin saberlo. Entre ellos se encuentran los estudios relacionados con el apego, inteligencia emocional, optimismo, conductas prosociales, valores humanos, creatividad, etc.; que se enmarcan dentro de los temas del campo de estudio de la psicología positiva. El mérito que corresponde a la Psicología Positiva es haber integrado en un corpus teórico pero con amplia validación empírica los mencionados tópicos de interés. (Lupano y Castro, 2010).

Se señala al año 1998 como el inicio de la Psicología positiva, con el discurso inaugural de Martin Seligman como presidente de la Asociación Psicológica Americana; el cual propuso el término indicando que su mandato tendría como misión enfatizar el interés hacia una psicología "más positiva". Es decir, retomar los objetivos olvidados de esta ciencia tradicionalmente centrada solo en curar la enfermedad: fortalecer y hacer más productiva la vida de las personas normales y promover la actualización del potencial humano (Gancedo, 2008). Para Seligman "la psicología no es solo el estudio de la debilidad y daño, es también el estudio de la fortaleza y virtud. El tratamiento no es solo arreglar lo que está roto, es también alimentar lo mejor de nosotros." (Seligman, 1998, p.18).

En un primer momento, Seligman considero que el énfasis debía estar en la prevención pero prontamente se dio cuenta que, ese modo, seguía enfocado en la enfermedad ya que el objetivo era meramente evitarla o minimizar sus efectos. (Seligman, 2003, p.1). 
Como hemos referido en líneas anteriores la preocupación y el interés por el estudio del bienestar humano y los factores que contribuyen al mismo, no es exclusividad de la psicología positiva. Sin embargo, Seligman tuvo la virtud de identificar, estas tendencias, nombrarlas y adaptarlas a los canones ortodoxos de la ciencia, organizada en una estructura programática, e impulsar su investigación y difusión (Gancedo, 2008; Lupano y Castro Solano).

Últimamente, se ha venido produciendo un cambio en la investigación en psicología, que evidencia una tendencia a abordar las variables positivas y preventivas en lugar de los aspectos negativos y patológicos que tradicionalmente se estudian (Guerrero y Vera, 2003; Simonton y Baumeister, 2005). Algunos autores sostienen que uno de los retos para la psicología en los próximos años será dedicar más trabajo intelectual al estudio de los aspectos positivos de la experiencia humana, entender y fortalecer aquellos factores que permiten prosperar a los individuos, comunidades y sociedades, para mejorar la calidad de vida y también prevenir las patologías que surgen de condiciones de vida adversas (Seligman y Csikszentmihalyi, 2000). El objetivo de lo que se ha llamado Psicología Positiva es justamente catalizar este cambio de la psicología, hacia el desarrollo de las fortalezas de las personas. Acorde con lo anterior, la principal tarea de prevención en esta década, será estudiar y entender cómo se adoptan esas fortalezas y virtudes en niños y jóvenes, elemento fundamental para la prevención de los llamados desórdenes mentales (Seligman y Christopher, 2000).

Según Seligman (1999), la psicología positiva surge como un intento de superar la resistente barrera del $65 \%$ de éxito que todas las psicoterapias han sido incapaces de sobrepasar hasta hoy. Las técnicas que surgen de la investigación en psicología positiva vienen a apoyar y complementar las ya existentes. Gracias a la investigación teórica en torno a esta área, el abanico de la intervención se verá ampliamente enriquecido. En este sentido, la relación de variables como el optimismo, el humor o las emociones positivas en los estados físicos de salud se alza como uno de los puntos clave de la investigación en psicología positiva.

\section{Conceptualización de la psicología positiva}

La psicología positiva se define como el estudio científico de las experiencias positivas, los rasgos individuales positivos, las instituciones que facilitan su desarrollo y los programas que ayudan a mejorar la calidad de vida de los individuos, mientras previene o reduce la incidencia de la psicopatología (Seligman 2005; Seligman y Csikszentmihalyi, 2000). Es definida también como el estudio científico de las fortalezas y virtudes humanas, las cuales permiten adoptar una perspectiva más abierta respecto al potencial humano, sus motivaciones y capacidades (Sheldon y King, 2001), incluye también virtudes cívicas e institucionales que guían a los individuos a tomar responsabilidades sobre su comunidad y promueve características para ser un mejor ciudadano. 
Dr. Luis Rodríguez de Los Ríos

A continuación presentaremos un cuadro de conceptualizaciones de la psicología positiva:

\begin{tabular}{|c|c|}
\hline Seligman y Christopher (2000) & $\begin{array}{l}\text { La psicología positiva es aquella que hace énfasis en actitudes } \\
\text { y emociones positivas, en potenciar fortalezas y fomentar } \\
\text { comportamientos que garanticen una buena calidad de vida. }\end{array}$ \\
\hline Sheldon y King (2001) & $\begin{array}{l}\text { La psicología positiva es una rama de la psicología que busca } \\
\text { comprender, a través de la investigación científica, los procesos } \\
\text { que subyacen a las cualidades y emociones positivas del ser } \\
\text { humano, durante tanto tiempo ignoradas por la psicología. }\end{array}$ \\
\hline Gable y Haidt (2005) & $\begin{array}{l}\text { La psicología positiva es el estudio de las condiciones y procesos } \\
\text { que contribuyen a prosperar o, al óptimo funcionamiento de } \\
\text { personas grupos e instituciones. }\end{array}$ \\
\hline $\begin{array}{l}\text { The Journal of Positive } \\
\text { Psychology (2005) }\end{array}$ & $\begin{array}{l}\text { La psicología positiva refiere a las perspectivas acerca de } \\
\text { que hace que la vida sea digna de ser vivida Se centra en los } \\
\text { aspectos de la condición humana que llevan a la felicidad, a la } \\
\text { completud y prosperar. }\end{array}$ \\
\hline $\begin{array}{l}\text { Linley, Joseph, Harrington y } \\
\text { Wood (2006) }\end{array}$ & $\begin{array}{l}\text { La psicología positiva es el estudio científico de las posibilidades } \\
\text { de un desarrollo humano óptimo: a nivel metapsicológico se } \\
\text { propone reorientar y reestructurar teóricamente el desequilibrio } \\
\text { existente en las investigaciones y prácticas psicológicas, } \\
\text { dando mayor relevancia al estudio de los aspectos positivos } \\
\text { de las experiencias vitales de los seres humanos. }\end{array}$ \\
\hline Carr (2007) & $\begin{array}{l}\text { La definió como un grupo de perspectivas científicas acerca } \\
\text { de lo que hace que la vida sea digna de ser vivida, que se } \\
\text { centra en los aspectos de la condición humana que llevan a la } \\
\text { felicidad, a la plenitud y a prosperar. }\end{array}$ \\
\hline Vera Poseck (2008) & $\begin{array}{l}\text { La psicología es una rama de la psicología que pretende a } \\
\text { través de la investigación científica, los procesos que subyacen } \\
\text { a las cualidades y emociones positivas del ser humano, durante } \\
\text { tanto tiempo ignoradas por la psicología. El objeto de este } \\
\text { interés es aportar nuevos conocimientos acerca de la psique } \\
\text { humana para alcanzar mejor calidad de vida y bienestar. }\end{array}$ \\
\hline Park y Peterson (2009) & $\begin{array}{l}\text { La psicología positiva es un nuevo enfoque de la psicología } \\
\text { que estudia lo que hace que la vida merezca ser vivida, es } \\
\text { el análisis de lo que va bien en la vida, desde el nacimiento } \\
\text { hasta la muerte. Dicho enfoque estudia la experiencia óptima, } \\
\text { esto es, a las personas siendo y dando lo mejor de sí mismas. }\end{array}$ \\
\hline
\end{tabular}

Fuente: Elaboración propia 


\section{Objetivos de la psicología positiva}

La psicología positiva tiene como objetivos mejorar la calidad de vida y prevenir la aparición de trastornos mentales y patologías. La concepción actual focalizada en lo patológico se centra en corregir defectos y reparar aquello que ya se ha roto. Por el contrario, la psicología positiva insiste en la construcción de competencias y en la prevención. (Seligman y Csikszentmihalyi, 2000). Esta ciencia y práctica, pretende poder prevenir muchos de los trastornos emocionales fundamentales y reorientar la psicología en sus dos vertientes relegadas: hacer más fuertes y productivas a las personas normales y hacer real el elevado potencial humano (Seligman, 2005).

Gancedo (2008) sintetiza la psicología positiva en los siguientes objetivos:

Figura 1

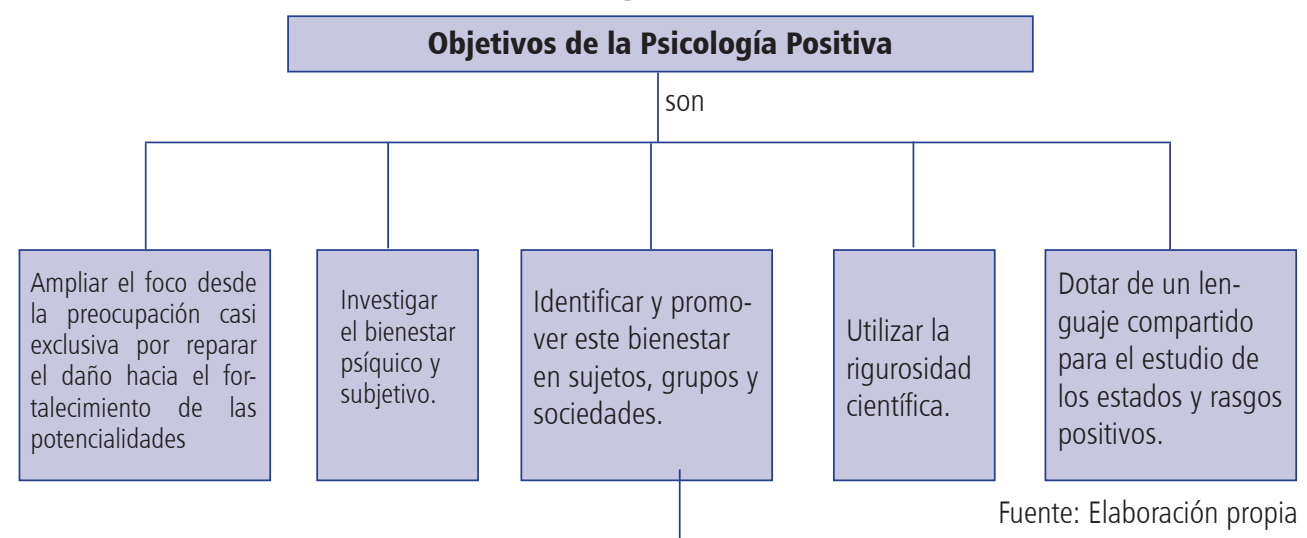

\section{Pilares básicos de la psicología positiva}

De acuerdo con Seligman (2002), los tres pilares básicos de estudio de la psicología positiva son: las emociones positivas, los rasgos positivos (virtudes y fortalezas personales) y las instituciones positivas que facilitan el desarrollo de dichas emociones y rasgos.

5.1. Emociones positivas: Emociones tales como la alegría, la esperanza, la ilusión, el agradecimiento, etc., cuyo valor adaptativo resulta fundamental para la especie humana, llevan a conectar con el primer tipo de felicidad, llamada "vida agradable" (pleasant life) que se refiere al bienestar que nos asalta al disfrutar de la buena comida, sexo, bebida e incluso una buena película. Este tipo de felicidad es de corta duración y se logra si conseguimos maximizar las emociones positivas, minimizando a su vez las negativas.

- Son ejemplo de emociones positivas del pasado: la satisfacción, la complacencia, la realización personal, el orgullo y la serenidad.

- Las emociones del presente son, entre otra: la alegría, el éxtasis, la tranquilidad, el entusiasmo, la euforia, el placer, la elevación y la fluidez.

- Finalmente, son emociones positivas del futuro: el optimismo, la esperanza, la fe y la confianza 
5.2. Rasgos positivos: Si se quiere alcanzar una vida satisfactoria, es necesario conocer y desarrollar aquellos rasgos de personalidad que nos ayuden a ser mejores y más felices. Con el objetivo de reconocer, medir y potenciar estos rasgos, se creó una clasificación de fortalezas y virtudes personales que se pueden identificar y promover para que las personas puedan alcanzar mayores niveles de bienestar. A través de estas fortalezas y virtudes llegamos al segundo tipo de felicidad, que se denomina "buena vida" (good life), la cual se desarrolla en cada uno cuando se disfruta haciendo algo en lo que se es bueno o se tiene talento, con lo que hay que tratar de identificarlas, para así poder potenciarlas, e incrementar nuestra "buena vida". (Seligman, 2003).

Seligman presentó una revisión de su teoría de la felicidad en su último libro "Flourish" (2011), traducido al español como "la vida que florece". En su teoría Seligman considera que el núcleo de la Psicología Educativa pasa a ser el bienestar en lugar que la felicidad; y que el patrón de medida y el objetivo dejan de ser la satisfacción con la vida para ser ahora el crecimiento personal, de esta manera, el constructo bienestar en esta nueva teoría, consta de cinco elementos que forman el acrónimo PERMA:

- Emociones Positivas (Positive Emotions): Cuanto mayor sea el número de emociones positivas que sintamos, mayor será nuestro bienestar.

- Entrega (Engagement): Es la capacidad de involucrarse de manera positiva en actividades del día a día y llegar a un estado conocido como flujo de conciencia (flow).

- Relaciones (Relationship): Cuantas más relaciones mantengamos a nuestro alrededor (amigos, conocidos, etc.), más bienestar sentiremos.

- Significado (Meaning): Entendido como la pertenencia a algo más grande que uno mismo, repercutirá en un mayor bienestar al tener un sentido vital.

- Logro (Accomplishment): Referido al sentimiento de valía, de sentir que se pueden hacer las cosas que se plantean, cumplir las metas.

\section{Figura 2}

\section{SELIGMAN, FLOURISH 2011}

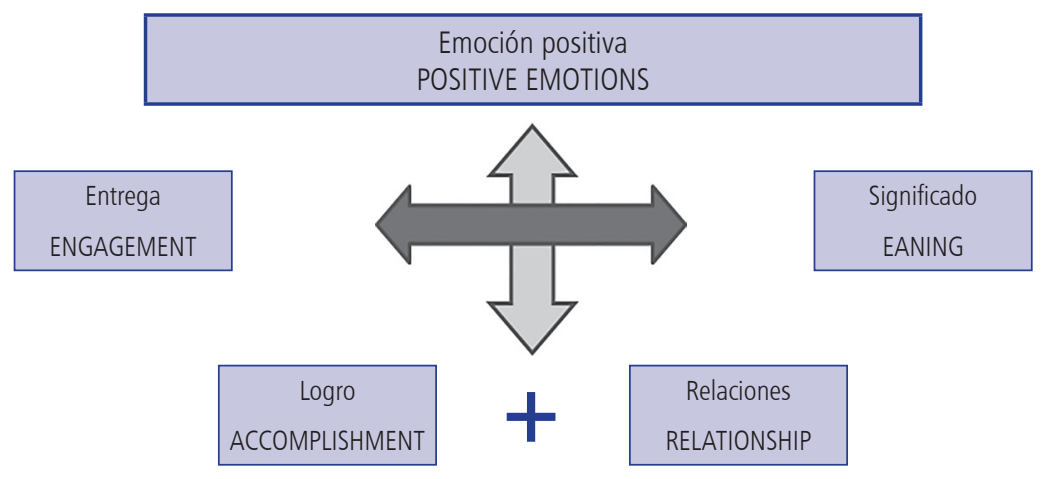

Modelo PERMA del bienestar de Seligman (2011) 
5.3. Instituciones positivas: Las sociedades actuales deben aspirar a poseer un conjunto de instituciones positivas que apoyen, fomenten y validen las emociones positivas y las fortalezas personales. Instituciones positivas son, por ejemplo, la familia, la libertad de información, la educación, etc., y aquí es donde se encuentra el tercer tipo de felicidad, denominado "vida con sentido" (meaningful life), que implica sentirse parte de estas instituciones. Es la más duradera de las tres y se trata de encontrar aquello en lo que creemos y de poner todas nuestras fuerzas a su servicio.

\section{Fuerzas o fortalezas humanas}

Peterson y Seligman (2004) hablan de "fuerzas o fortalezas humanas" (strengthsof caracter) para referirse a las características, manifestaciones psicológicas o recursos positivos de la persona. Tras sus investigaciones, aunque existan muchos matices y discrepancias en torno a lo que constituye el "buen carácter", se hallaron seis fortalezas positivas generales que podrían encontrarse en todas las culturas, denominados virtudes. A su vez cada una de ellas se descompone en fortalezas. Las seis virtudes serían: Sabiduría y conocimiento, coraje, humanidad, justicia, templanza y trascendencia. Ver Tabla 1.

\section{Tabla 1}

\begin{tabular}{|c|c|c|c|c|c|}
\hline \multicolumn{6}{|c|}{ VIRTUDES Y FORTALEZAS } \\
\hline SABIDURIA & CORAJE & HUMANIDAD & JUSTICIA & TEMPLANZA & TRANSCENDENCIA \\
\hline $\begin{array}{l}\text { Creatividad } \\
\text { Curiosidad } \\
\text { Apertura } \\
\text { mental Deseo } \\
\text { de aprender } \\
\text { Perspectiva } \\
\text { (Sabiduría) }\end{array}$ & $\begin{array}{l}\text { Valentía } \\
\text { Persistencia } \\
\text { Integridad } \\
\text { Vitalidad }\end{array}$ & $\begin{array}{l}\text { Amor } \\
\text { Amabilidad } \\
\text { Inteligencia } \\
\text { social }\end{array}$ & $\begin{array}{l}\text { Ciudadanía } \\
\text { Equidad } \\
\text { Liderazgo }\end{array}$ & $\begin{array}{l}\text { Capacidad } \\
\text { de perdón } \\
\text { Humildad } \\
\text { Prudencia Auto } \\
\text { - regulación }\end{array}$ & $\begin{array}{l}\text { Capacidad estética } \\
\text { Gratitud Esperanza } \\
\text { Humor Espiritualidad }\end{array}$ \\
\hline
\end{tabular}

Virtudes y fortalezas (Peterson y Seligman, 2004)

\section{Campos de aplicación de la psicología positiva}

Dado que el propósito de la psicología positiva ha sido contribuir al estudio de las condiciones y procesos relacionados con el desarrollo óptimo de los individuos, grupos e instituciones (Gable y Haidt, 2005), sus aportes han tenido impacto en distintos campos de intervención de la psicología, principalmente en las áreas clínica, de la salud y educativa, en las que se observa un mayor énfasis.

Desde la educación la psicología positiva centra su atención en las fortalezas o atributos específicos positivos de las personas y grupos en ambientes pedagógicos (Hughes, 2000). De acuerdo con estudios realizados en este ámbito, aquellos docentes que enfatizan en las experiencias positivas sobre el desarrollo de habilidades en los niños, hacen que éstos tengan mayor probabilidad de experimentar altos niveles de autoeficacia y otras características de desarrollo psicológico positivo, cuando ocurren conjuntamente con el desarrollo de habilidades complejas (Akin-Little, Little y Delligatti, 2004). Al respecto, Seligman y Csikszentmihalyi (2000) sostienen que promover 
competencias en los niños es más que arreglar lo que está mal en ellos; es identificar y fortalecer sus cualidades predominantes, y ayudarles a encontrar los espacios en los que puedan expresarlas. Promover las fortalezas de los niños y jóvenes puede incrementar cambios que favorecen el manejo exitoso de las dificultades actuales y las que deberán afrontar en el futuro (Terjesen, Jacofsky, Froh y DiGiuseppe, 2004).En este sentido, Meyers y Nastasy (1999), sostienen que las intervenciones preventivas deben dirigirse hacia la modificación del ambiente para reducir el estrés, modificación del individuo para desarrollar competencias, a modificar de forma simultánea al individuo y su ambiente. Los ambientes educativos en los cuales las recompensas son ofrecidas de manera contingente con el logro exitoso de metas realistas tienen más probabilidad de incrementar la motivación y disminuir los comportamientos problemáticos de los niños y jóvenes (Jenson, Olympia, Farley y Clark, 2004).

\section{Intervención en el ámbito educativo}

\subsection{Programa Aulas felices}

Es un programa educativo basado en la Psicología Positiva. Este movimiento persigue conseguir que el foco de atención de la Psicología se centre no sólo en reparar las peores cosas de la vida, sino contribuir a la construcción de cualidades positivas (Seligman y Csikszentmihalyi, 2000). Durante los últimos años, la Psicología Positiva ha avanzado considerablemente en el conocimiento sobre la felicidad humana y el desarrollo de las fortalezas personales, y aunque desde un principio Seligman insistió en la importancia de la familia y de la escuela como origen de estas fortalezas, han sido pocos, hasta hoy, los trabajos que han intentado incorporar los contenidos de esta orientación psicológica al campo de la educación. En este sentido, Programa AULAS FELICES es una apuesta importante y valiosa. Los objetivos del programa son potenciar el desarrollo personal y social del alumnado y promover la felicidad de los alumnos, los profesores y las familias. Ver tabla 2. Para ello, ofrece reflexiones, técnicas y recursos que pretenden unificar y estructurar el trabajo que en la actualidad se está desarrollando en las escuela en torno a la Acción Tutorial, la Educación en Valores y las Competencias Básicas, relacionadas con el desarrollo personal, social y aprender a aprender. Los autores parten de la idea de que el objetivo del maestro es capacitar a los niños y jóvenes para desplegar al máximo sus aspectos positivos y potenciar su bienestar presente y futuro. Consideran que no es necesario llegar a ser adulto para ser feliz con los aprendizajes de la infancia o que tener un trabajo en el futuro no conlleva necesariamente satisfacción si antes no nos han enseñado a disfrutar de lo que somos, en definitiva, que se puede ser feliz en el aula. En la base está el principio pedagógico de promover una educación en la que prime más la calidad que la cantidad. El programa incorpora referencias a diferentes modelos y estrategias de enseñanza aprendizaje que comparten la filosofía de la Psicología Positiva (socioconstructivismo, aprendizaje cooperativo), así como a programas ya existentes que pueden ayudar a desarrollar las diferentes fortalezas personales. 
Tabla 2

\begin{tabular}{|c|c|c|c|c|}
\hline Objetivos & $\begin{array}{c}\text { Componentes del } \\
\text { Programa }\end{array}$ & $\begin{array}{l}\text { Elementos del } \\
\text { currículo educativo } \\
\text { en los que se integra }\end{array}$ & $\begin{array}{l}\text { Niveles de } \\
\text { intervención }\end{array}$ & $\begin{array}{c}\text { Alumnado al que } \\
\text { se dirige }\end{array}$ \\
\hline $\begin{array}{l}\text {-Potenciar el desarrollo } \\
\text { personal y social del } \\
\text { alumnado. } \\
\text {-Promover la felicidad } \\
\text { de los alumnos, } \\
\text { los profesores y las } \\
\text { familias. }\end{array}$ & $\begin{array}{l}\text { 1. La atención plena } \\
\text { ("mindfulness"). } \\
\text { 2. La educación de las } \\
24 \text { fortalezas personales } \\
\text { (Peterson y Seligman, 2004): } \\
\text { Propuestas globales. } \\
\text {.Propuestas específicas. } \\
\text {.Planes personalizados. }\end{array}$ & $\begin{array}{l}\text {-.Trabajo conjunto } \\
\text { entre familias y centros } \\
\text { educativos. } \\
\text {-Competencias Básicas: } \\
\text { sentido de iniciativa y } \\
\text { espíritu emprendedor, } \\
\text { sociales y cívicas, y } \\
\text { aprender a aprender. } \\
\text {-Acción Tutorial. } \\
\text {-Educación en Valores }\end{array}$ & $\begin{array}{l}\text {-Las aulas. } \\
\text {-Los Equipos } \\
\text { de Ciclo o } \\
\text { Departamentos. } \\
\text {-La Institución } \\
\text { Educativa. } \\
\text {-La Comunidad } \\
\text { Educativa. }\end{array}$ & $\begin{array}{l}\text { - Es t u d i a n t es } \\
\text { de Educación } \\
\text { Infantil, Primaria y } \\
\text { Secundaria. }\end{array}$ \\
\hline
\end{tabular}

Modelo Aulas Felices (Seligman y Csikszentmihalyi, 2000)

\section{Finalidad del programa}

Se pretende potenciar aprendizajes y felicidad en estudiantes:

- La Atención Plena les ayuda a vivir conscientemente, y a disfrutar y gobernar mejor su vida.

- Las Fortalezas Personales les permiten cultivar su vida interior, para que la felicidad dependa de sí mismos y no tanto de lo exterior.

- Desarrollando la atención plena y las fortalezas personales, lograremos potenciar los 5 componentes básicos de la felicidad (emociones positivas, compromiso, significado, relaciones y logros), que favorecerán el bienestar y los aprendizajes.

Desarrollo de las 24 fortalezas personales:

SABIDURÍA Y CONOCIMIENTO: Fortalezas cognitivas que implican la adquisición y el uso del conocimiento:

\begin{tabular}{|c|c|c|c|c|}
\hline $\begin{array}{l}\text { 1.Creatividad } \\
\text { (originalidad, } \\
\text { ingenio). }\end{array}$ & $\begin{array}{l}\text { 2. Curiosidad } \\
\text { (interés por el } \\
\text { mundo, búsqueda } \\
\text { de novedades, } \\
\text { apertura a } \\
\text { experiencias) }\end{array}$ & $\begin{array}{l}\text { 3. Apertura mental } \\
\text { (juicio, pensamiento } \\
\text { crítico) }\end{array}$ & $\begin{array}{l}\text { 4. Amor por el } \\
\text { aprendizaje. }\end{array}$ & $\begin{array}{l}\text { 5.Perspectiva } \\
\text { (sabiduría). }\end{array}$ \\
\hline $\begin{array}{l}\text { Pensar en nuevos y } \\
\text { productivos modos } \\
\text { de conceptualizar } \\
\text { y hacer las } \\
\text { cosas. Incluye la } \\
\text { creación artística } \\
\text { pero no se limita } \\
\text { exclusivamente a } \\
\text { ella. }\end{array}$ & $\begin{array}{l}\text { Tener interés por lo } \\
\text { que sucede en el } \\
\text { mundo, encontrar } \\
\text { temas fascinantes, } \\
\text { explorar y descubrir } \\
\text { nuevas cosas. }\end{array}$ & $\begin{array}{l}\text { Pensar sobre las cosas } \\
\text { y examinar todos sus } \\
\text { significados y matices. } \\
\text { No precipitarse al sacar } \\
\text { conclusiones, sino tras } \\
\text { evaluar cada posibilidad. } \\
\text { Ser capaz de cambiar las } \\
\text { propias ideas en base a } \\
\text { la evidencia. Ponderar } \\
\text { adecuadamente cada } \\
\text { evidencia. }\end{array}$ & $\begin{array}{l}\text { Llegar a dominar nuevas } \\
\text { habilidades, materias y } \\
\text { conocimientos, ya sea por } \\
\text { sí mismo o mediante un } \\
\text { aprendizaje formal. Está } \\
\text { obviamente relacionada } \\
\text { con la fortaleza de } \\
\text { curiosidad pero va más } \\
\text { allá, describiendo una } \\
\text { tendencia sistemática a } \\
\text { añadir cosas a lo que uno } \\
\text { sabe. }\end{array}$ & $\begin{array}{l}\text { Ser capaz de dar } \\
\text { consejos sabios } \\
\text { y adecuados } \\
\text { a los demás, } \\
\text { encontrando modos } \\
\text { para comprender } \\
\text { el mundo y } \\
\text { para ayudar a } \\
\text { comprenderlo a los } \\
\text { demás. }\end{array}$ \\
\hline
\end{tabular}




\section{CORAJE: Fortalezas emocionales que implican el ejercicio de la voluntad para la consecución de metas ante situaciones de dificultad, externa o interna:}

\begin{tabular}{|c|c|c|c|}
\hline 6. Valentía (valor). & $\begin{array}{c}\text { Perseverancia } \\
\text { (tenacidad, diligencia, } \\
\text { laboriosidad). }\end{array}$ & $\begin{array}{l}\text { 8. Integridad } \\
\text { (autenticidad, } \\
\text { honestidad). }\end{array}$ & $\begin{array}{c}\text { 9. Vitalidad (ánimo, } \\
\text { entusiasmo, vigor, } \\
\text { energía) }\end{array}$ \\
\hline $\begin{array}{l}\text { No dejarse intimidar ante } \\
\text { la amenaza, el cambio, la } \\
\text { dificultad o el dolor. Ser capaz } \\
\text { de defender una postura que } \\
\text { uno cree correcta aunque } \\
\text { exista una fuerte oposición } \\
\text { por parte de los demás. } \\
\text { Actuar según las propias } \\
\text { convicciones aunque eso } \\
\text { suponga ser criticado. Incluye } \\
\text { el valor físico pero no se } \\
\text { limita a él. }\end{array}$ & $\begin{array}{l}\text { Terminar lo que uno empieza. } \\
\text { Persistir en una actividad } \\
\text { aunque existan obstáculos. } \\
\text { Obtener satisfacción por las } \\
\text { tareas emprendidas y que } \\
\text { consiguen finalizarse con } \\
\text { éxito. }\end{array}$ & $\begin{array}{l}\text { Ir siempre con la verdad por } \\
\text { delante, presentarse ante los } \\
\text { demás de un modo genuino } \\
\text { y actuando con sinceridad; } \\
\text { no ser pretencioso; asumir } \\
\text { la responsabilidad de los } \\
\text { propios sntimientos y } \\
\text { acciones. }\end{array}$ & $\begin{array}{l}\text { Afrontar la vida con } \\
\text { entusiasmo y energía; hacer } \\
\text { las cosas con convicción } \\
\text { y dando todo de uno } \\
\text { mismo; vivir la vida como } \\
\text { una apasionante aventura; } \\
\text { sentirse vivo y activo. }\end{array}$ \\
\hline
\end{tabular}

HUMANIDAD: Fortalezas interpersonales que implican cuidar y ofrecer amistad y cariño a los demás:

\begin{tabular}{|l|l|l|}
\hline $\begin{array}{c}\text { 10. Amor (capacidad de amar y } \\
\text { ser amado). }\end{array}$ & $\begin{array}{c}\text { 11. Amabilidad (bondad, } \\
\text { generosidad, cuidado, } \\
\text { compasión, amor altruista, } \\
\text { simpatía). }\end{array}$ & $\begin{array}{c}\text { 12. Inteligencia social } \\
\text { (inteligencia emocional, } \\
\text { inteligencia personal). }\end{array}$ \\
\hline $\begin{array}{l}\text { Valorar las relaciones cercanas con otras } \\
\text { personas, en particular aquellas en las } \\
\text { que el afecto y el cuidado son mutuos. } \\
\text { Sentirse cercano a otras personas. }\end{array}$ & $\begin{array}{l}\text { Hacer favores y buenas acciones para } \\
\text { los demás, ayudar y cuidar a otras } \\
\text { personas. }\end{array}$ & $\begin{array}{l}\text { Ser consciente de las motivaciones y los } \\
\text { sentimientos, tanto de uno mismo como } \\
\text { de los demás; saber cómo comportarse } \\
\text { en las diferentes situaciones sociales; } \\
\text { saber qué cosas son importantes para } \\
\text { otras personas, tener empatía. }\end{array}$ \\
\hline
\end{tabular}

\section{JUSTICIA: Fortalezas cívicas que conllevan una vida en comunidad saludable:}

\begin{tabular}{l|l|l|}
$\begin{array}{l}\text { 13. Ciudadanía (responsabilidad } \\
\text { social, lealtad, trabajo en } \\
\text { equipo). }\end{array}$ & \multicolumn{1}{|c|}{ 14. Sentido de la justicia. } & \multicolumn{1}{c|}{ 15. Liderazgo. } \\
\hline $\begin{array}{l}\text { Trabajar bien dentro de un equipo o } \\
\text { grupo de personas; ser fiel al grupo; } \\
\text { cumplir las tareas asignadas dentro de } \\
\text { él. }\end{array}$ & $\begin{array}{l}\text { Tratar a todas las personas como iguales } \\
\text { en consonancia con las nociones de } \\
\text { equidad y justicia; no dejar que los } \\
\text { sentimientos personales influyan en las } \\
\text { decisiones sobre los otros; dar a todo el } \\
\text { mundo las mismas oportunidades. }\end{array}$ & $\begin{array}{l}\text { Animaral grupo del que uno es miembro } \\
\text { para hacer cosas, así como reforzar las } \\
\text { relaciones entre las personas de dicho } \\
\text { procurar llevarlas a buen término. }\end{array}$ \\
\hline
\end{tabular}




\section{MODERACIÓN: Fortalezas que nos protegen contra los excesos:}

\begin{tabular}{|c|c|c|c|}
\hline $\begin{array}{l}\text { 16. Capacidad de } \\
\text { perdonar, misericordia. }\end{array}$ & $\begin{array}{l}\text { 17. Modestia, } \\
\text { humildad. }\end{array}$ & $\begin{array}{l}\text { 18. Prudencia } \\
\text { (discreción, cautela). }\end{array}$ & $\begin{array}{l}\text { 19. Autocontrol, } \\
\text { autorregulación. }\end{array}$ \\
\hline $\begin{array}{l}\text { Capacidad de perdonar a } \\
\text { aquellas personas que han } \\
\text { actuado mal; aceptar los } \\
\text { defectos de los demás; dar } \\
\text { a los otros una segunda } \\
\text { oportunidad; no ser } \\
\text { vengativo ni rencoroso. }\end{array}$ & $\begin{array}{l}\text { Dejar que los propios } \\
\text { logros hablen por sí } \\
\text { mismos; no buscar ser el } \\
\text { centro de atención; no } \\
\text { considerarse más especial } \\
\text { de lo que uno es en } \\
\text { realidad. }\end{array}$ & $\begin{array}{l}\text { Ser cauteloso a la hora de } \\
\text { tomar decisiones; no asumir } \\
\text { riesgos innecesarios; no } \\
\text { decir ni hacer nada de lo } \\
\text { que después uno se pueda } \\
\text { arrepentir. }\end{array}$ & $\begin{array}{l}\text { Tener capacidad para regular } \\
\text { los propios sentimientos y } \\
\text { acciones; ser disciplinado; } \\
\text { controlar los propios impulsos } \\
\text { y emociones. }\end{array}$ \\
\hline
\end{tabular}

\section{TRASCENDENCIA: Fortalezas que forjan conexiones con la inmensidad del universo y proveen de significado a la vida:}

\begin{tabular}{|c|c|c|c|c|}
\hline $\begin{array}{l}\text { 20. Apreciación } \\
\text { de la belleza y } \\
\text { la excelencia } \\
\text { [admiración, } \\
\text { asombro]. }\end{array}$ & 21. Gratitud. & $\begin{array}{l}\text { 22. Esperanza } \\
\text { (optimismo, } \\
\text { proyección hacia } \\
\text { el futuro). }\end{array}$ & $\begin{array}{l}\text { 23. Sentido del } \\
\text { humor (capacidad } \\
\text { de diversión). }\end{array}$ & $\begin{array}{c}\text { 24. Espiritualidad } \\
\text { (sentido religioso, } \\
\text { fe, sentido en la } \\
\text { vida). }\end{array}$ \\
\hline $\begin{array}{l}\text { Saber reconocer y } \\
\text { apreciar la belleza, } \\
\text { la excelencia y las } \\
\text { cosas bien hechas } \\
\text { en variados ámbitos } \\
\text { de la vida como } \\
\text { la naturaleza, el } \\
\text { arte, la ciencia, } \\
\text { las experiencias } \\
\text { cotidianas.. }\end{array}$ & $\begin{array}{l}\text { Ser consciente de y } \\
\text { agradecer las cosas } \\
\text { buenas que suceden; } \\
\text { saber expresar } \\
\text { agradecimiento. }\end{array}$ & $\begin{array}{l}\text { Esperar lo mejor para } \\
\text { el futuro y trabajar para } \\
\text { conseguirlo; creer que } \\
\text { un buen futuro es algo } \\
\text { que está en nuestras } \\
\text { manos conseguir. }\end{array}$ & $\begin{array}{l}\text { Gustar de reír y } \\
\text { bromear; sonreír con } \\
\text { frecuencia; ver el lado } \\
\text { positivo de las cosas. }\end{array}$ & $\begin{array}{l}\text { Pensar que existe } \\
\text { un propósito o un } \\
\text { significado universal } \\
\text { en las cosas que } \\
\text { ocurren en el mundo y } \\
\text { en la propia existencia; } \\
\text { sentir que tu propia } \\
\text { existencia se inserta } \\
\text { en un marco más } \\
\text { amplio de significados; } \\
\text { tener creencias acerca } \\
\text { del significado de la } \\
\text { vida que dan forma a } \\
\text { nuestra conducta y nos } \\
\text { confortan. }\end{array}$ \\
\hline
\end{tabular}

\subsection{El Mindfulness}

El Mindfulness puede considerarse una filosofía de vida que incluye la práctica de la meditación. Juntamente a varias técnicas de relajación, su apogeo es reciente. Mindfulness, literalmente atención o conciencia plena, es una de las múltiples formas de meditación que se basa en centrar la mente en el momento presente, es decir, es una conciencia que se desarrolla prestando una atención concreta, sostenida y deliberada sin juzgar las experiencias del aquí y del ahora (KabatZinn, 2013).

Ya hace mucho tiempo que se incorporaron con éxito los programas terapéuticos de reducción del estrés basados en el mindfulness (del inglés, MBSR) para sobrellevar el dolor crónico, aliviar el sufrimiento psicológico o mitigar la ansiedad y la depresión pero, en los últimos años, se han identificado los cambios cerebrales que producen este tipo de prácticas: 8 semanas de entrenamiento son suficientes para incrementar la actividad de la corteza prefrontal izquierda que está asociada 
al bienestar y la resiliencia (Davidson y Begley, 2012) o para aumentar la concentración de materia gris en regiones cerebrales que intervienen en procesos relacionados con la memorización y aprendizaje.

Por tanto, esta filosofía puede ser adaptada a diferentes contextos y entornos, porque su enfoque es pragmático y no depende de dogmas religiosos. Y, lo que es más importante, su popularidad ha hecho que se esté creando una biblioteca de literatura científica en la que se incluyen muchos estudios que exploran el potencial del Mindfulness en diferentes facetas: el autocontrol en los niños y niñas, el desarrollo de resiliencia y recursos de afrontamiento en personas enfermas, la mejora en niveles objetivos de salud, etc.

Aunque la mayoría de las investigaciones sobre los efectos de estas prácticas se habían realizado con adultos, ya disponemos en los últimos tiempos de estudios que demuestran los beneficios del mindfulness relacionados con la salud, el bienestar psicológico, las competencias sociales o el rendimiento académico de niños y adolescentes e, incluso, se ha analizado también la incidencia positiva sobre el estrés o el burnout en profesores. Y es que el mindfulness, al igual que el ejercicio físico, constituye una forma de actividad (en este caso mental) que promueve sus mismos beneficios. Y ello tiene grandes implicaciones educativas porque cuando los alumnos mejoran su capacidad atencional y se encuentran más relajados se facilita su aprendizaje.

\section{Objetivo del Mindfulness}

Mindfulness tiene el objetivo de mejorar la calidad de vida de las personas en unos términos muy concretos, y no están vinculadas a una religión o una filosofía de vida determinadas. Por lo tanto, la práctica del Mindfulness está desvinculada de creencias religiosas y filosofías de vida concretas; es, simplemente, una práctica que puede convertirse en herramienta para mejorar la calidad de vida de las personas de manera demostrable.

\section{Mindfulness para estudiantes}

En muchas investigaciones han sido reportados los beneficios de los programas de educación socioemocional. Así, por ejemplo, en un metaanálisis con más de 270000 alumnos de todas las etapas académicas, se demostró que aquellos que participaron en primaria en este tipo de programas

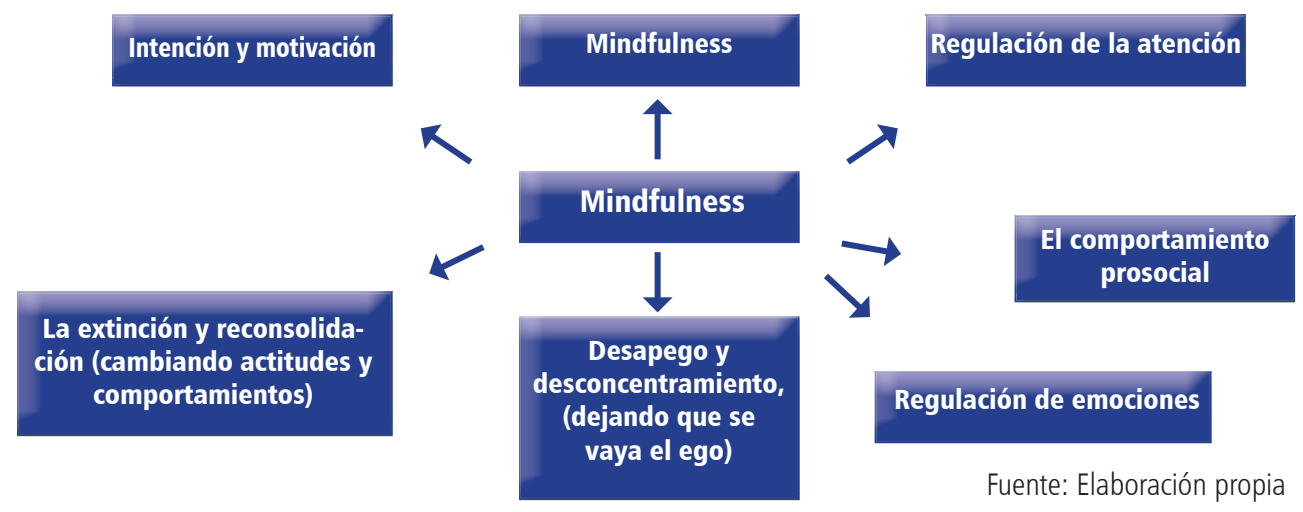


no solo mostraron mejoras significativas siendo adolescentes en cuestiones conductuales, sino que también obtuvieron una mejora en promedio del $11 \%$ en sus resultados académicos respecto a los que no recibieron esa formación (Durlak et al., 2011). Pero, además, cuando se añaden a este tipo de programas de educación socioemocional las prácticas contemplativas como el mindfulness se mejoran los resultados obtenidos en relación a cuando se utilizan estas técnicas por separado.

Por ejemplo, cuando un estudiante está alterado, decirle que tome conciencia de sus propias emociones puede ser insuficiente; o la simple práctica del mindfulness no garantiza que adquiera las competencias necesarias para resolver conflictos. Sin embargo, cuando se integra el mindfulness en los programas de educación socioemocional, algunas de sus competencias se ven reforzadas: la autoconciencia adopta una nueva profundidad de exploración interior, la gestión emocional fortalece la capacidad para resolver conflictos y la empatía se convierte en la base del altruismo y la compasión (Lantieri y Zakrzewski, 2015).

\subsection{La Cognición Instruccional Positiva (CIP)}

La Cognición Instruccional Positiva (CIP) es una propuesta teórico-práctica basada en la integralidad de la formación humana propuesta desde los organismos educativos mundiales y en los modelos psicológicos de autores como Frankl y Seligman. El paralelismo epistemológico entre estas teorías, tan solo separadas por algunas décadas, ha sido recientemente estudiado y evidenciado por algunos autores (Risco, 2009; Salvino, 2009).

Mostrando estos estudios resultados que evidencian la correspondencia entre la formulación de Seligman y los niveles vitales de la teoría de Frankl. La propuesta realizada por el proyecto DESECO (Simone y Herhs, 2001), patrocinado por la Organización de Cooperación y Desarrollo Económicos (OCDE) avala la formación competencial integral en la educación. Las características requeridas las encontramos en la tridimensionalidad competencial de la formación integral (Delors, 1996; Cabaco, 2009):

- Saber (capaz de centrar su atención y planear);

- Saber hacer (obrar de forma autosuficiente);

- Saber ser (sentido de responsabilidad y sentido de vida), y son desarrolladas en los cinco niveles en los que se concreta la proposición de la CIP (Cabaco, 2009).

Esta propuesta pretende ser una guía aplicada para el desarrollo del bienestar al que apuntan tanto el suprasentido de la teoría de Frankl, como la felicidad o vida plena de la Psicología Positiva de Seligman. Los niveles son los siguientes: Análisis del problema: supone un entrenamiento instruccional específico centrado en adquirir e interiorizar un esquema de afrontamiento y en la adquisición de un alto grado de supervisión metacognitivo hacia las propias intervenciones.

Para ello, se emplea $\mathrm{G}$ los patrones de los enfoques contemporáneos de la solución de problemas de corte cognitivo (Bruning, Schraw, Norby y Ronning, 2005), que proponen cuatro etapas en su procedimiento general:

a) Identificar el problema, supone definir el problema en términos específicos, operativos y resolubles. 
b) Representar el problema, que operativizamos mediante los cuatro ejes que globalizan el espacio del problema: estado inicial (identificación del problema), estado final o meta (situación que definirá la solución misma), operadores o estrategias a utilizar (recursos disponibles o posibles) y restricciones o limitaciones (hándicaps o problemas).

c) Seleccionar la estrategia, ya que cada problema puede tener distinta magnitud en cuanto a su complejidad, soluciones posibles (representaciones). Además es muy importante la discriminación de la estructura profunda y aplicar, por ejemplo, el razonamiento analógico para problemas que tengan la misma naturaleza estructural a pesar de las diferencias aparentes en su manifestación superficial.

d) Poner en práctica y evaluar, es decir, pasar de las competencias cognitivas (saber) a las procedimentales (saber hacer) pero con una actitud de autorregulación (saber ser). La experiencia indica que el éxito de una estrategia depende básicamente de los primeros pasos (adecuada identificación y representación de los problemas) pero también de la flexibilidad para cambiar de estrategia y evaluar de forma anticipada las consecuencias de su aplicación. Expresión y reconocimiento emocional: se busca potenciar la capacidad de expresión (función comunicativa hacia fuera) y de reconocimiento emocional (función comunicativa hacia dentro). Además, es muy importante el papel regulador que las emociones positivas pueden ejercer sobre las negativas desde el plano adaptativo y autorregulador de la conductas (Fernández-Abascal, 2008).

Para algunos autores este dominio emocional podría denominarse conciencia emocional (Iriarte, Alonso y Sabino, 2006), en la medida que su dominio permite conocer las emociones en el momento de su aparición (poder ejercer control), ser consciente de su funcionamiento (buscar idoneidad emocional), así como de las consecuencias (dimensiones adaptativas). Esta etapa se correspondería con lo expresado por Seligman como vida placentera (risas, buen humor, etc.) y como "cumplimiento de un sentido" y "encuentro humano" que aleja del vacío existencial, se pretende desarrollar lo siguiente:

- Autoconocimiento: aquí se pretende fortalecer a la persona contra las situaciones 0 contextos generadores de inestabilidad personal y social que se puedan presentar mediante estrategias instruccionales que favorezcan el autoconocimiento. De este autoconocimiento deriva lo que Seligman denomina el segundo nivel de felicidad: la vida comprometida, y la voluntad de sentido como la motivación esencial del comportamiento humano.

- Reflexión o contemplación global de la situación/problema y de su implicación en la dimensión personal. En este nivel es de gran utilidad la llamada inteligencia intuitiva (Gladwell, 2005), inspirada en las inteligencias múltiples de Gardner (1995). Este tipo de conducta inteligente permite diferenciar si la cognición rápida (selección de datos significativos en una situación contextual determinada) que una persona hace es objetiva o está "contaminada" por lastres o resonancias personales. Esta formulación tiene su fundamento en los trabajos de Wilson (2002) desde el plano cognitivo o en Damasio (2001) desde el plano neuropsicológico.

- Bienestar: aquí se busca alcanzar el nivel de felicidad correspondiente al concepto de vida significativa de Seligman, que propone una aplicación de los puntos fuertes personales con el objetivo de sentido de pertenencia y de servicio hacia algo que trasciende. 


\subsection{La experiencia de fluidez en la educación}

El fluir o flujo (en inglés flow) ha sido de gran utilidad para los psicólogos que estudian la felicidad, la satisfacción vital y la motivación intrínseca. Ha sido estudiado por Mihaly Csikszentmihalyi (1997), quien lo ha definido como un estado en el cual las personas se hallan tan involucradas en la actividad que nada más parece importarles; la experiencia, por sí misma, es tan placentera que las personas la realizarán incluso aunque implique un gran esfuerzo, por el puro placer de hacerla.

Las personas que experimentan fluidez sienten que controlan sus acciones y son dueñas de su destino, sienten júbilo y una profunda sensación de satisfacción, más allá del mero divertimento. La fluidez se produce cuando las habilidades de la persona están en equilibrio con los retos y desafíos que la actividad plantea. En estas circunstancias, la atención se centra en la consecución de una meta realista y la retroalimentación que se obtiene sobre el nivel de ejecución de manera inmediata. A partir de investigaciones con un grupo de artistas, estudiantes, deportistas, científicos y profesionales de distintas áreas.

Csikszentmihalyi estudió la variedad de experiencias que se llegó a conocer como flujo o fluir. La palabra fluir significa "seguir la corriente" y fue la elegida para describir esos momentos de calidad llamados experiencias óptimas. Lo que más le interesaba, era conocer la calidad de la experiencia subjetiva que determinaba una conducta intrínsecamente motivadora ¿Cómo se sentían las recompensas intrínsecas? ¿Por qué eran recompensantes? ¿Por qué algunas personas se concentran tanto en conseguir buenos resultados? ¿Cómo las metas y proyectos personales incentivan los logros?

La experiencia de fluir tiene como características obtener un lugar en casi cualquier actividad y parece ser un fenómeno que todo el mundo siente de la misma manera, sin tener en cuenta cuestiones de edad o sexo, nivel cultural o clase social. En términos muy similares independientemente del contexto hablan artistas, atletas, compositores, bailares, científicos, estudiantes y personas de todas las profesiones, cuando describen sus sentimientos realizando algo que merece la pena hacer (Caruana, 2010). Las condiciones que se deben dar para llegar a la experiencia de fluidez dependen según Csikszentmihalyi (1997, 1998), de:

1. Objetivos claros. Las personas intentan tener proyectos, metas claras y una retroalimentación constante.

2. Equilibrio entre desafío-habilidad. Las oportunidades para actuar de manera adecuada son relativamente altas y encajan con la propia habilidad percibida para actuar. En otras palabras, las habilidades personales se ajustan a los desafíos dados.

3. Fusión acción-atención. La acción y la atención se funden únicamente cuando se llega a estar totalmente concentrado en lo que se está haciendo. Esto sucede cuando se siente que tiene las posibilidades y habilidades para superar el desafío y cuando se centra toda la atención en la tarea. La conciencia unificada que acompaña a la fusión de acción y atención es quizás el aspecto más revelador de la experiencia. Las emociones positivas del fluir, pueden ser una fuente de motivación (retroalimentación), capaz de crear un mecanismo que permita volver de nuevo a este estado óptimo (acciónatención-meta)

4. La experiencia se torna autotélica: si algunas de las condiciones previas están presentes, lo que uno hace se convierte en autotélico, podría decirse auto gratificante, que vale la pena hacerlo por sí mismo. Las experiencias de fluidez (Csikszentmihalyi, 1998) pueden ser accesibles a todas las personas en todos los ámbitos del quehacer humano; casi toda actividad provechosa y útil (jugar, trabajar, estudiar, explorar) es capaz de producirlas, siempre que se hallen presentes algunas de las condiciones antes reseñadas. 
Según Csikszentmihalyi (1997), las razones que hacen que el fluir sea beneficioso son:

\section{Figura 3}

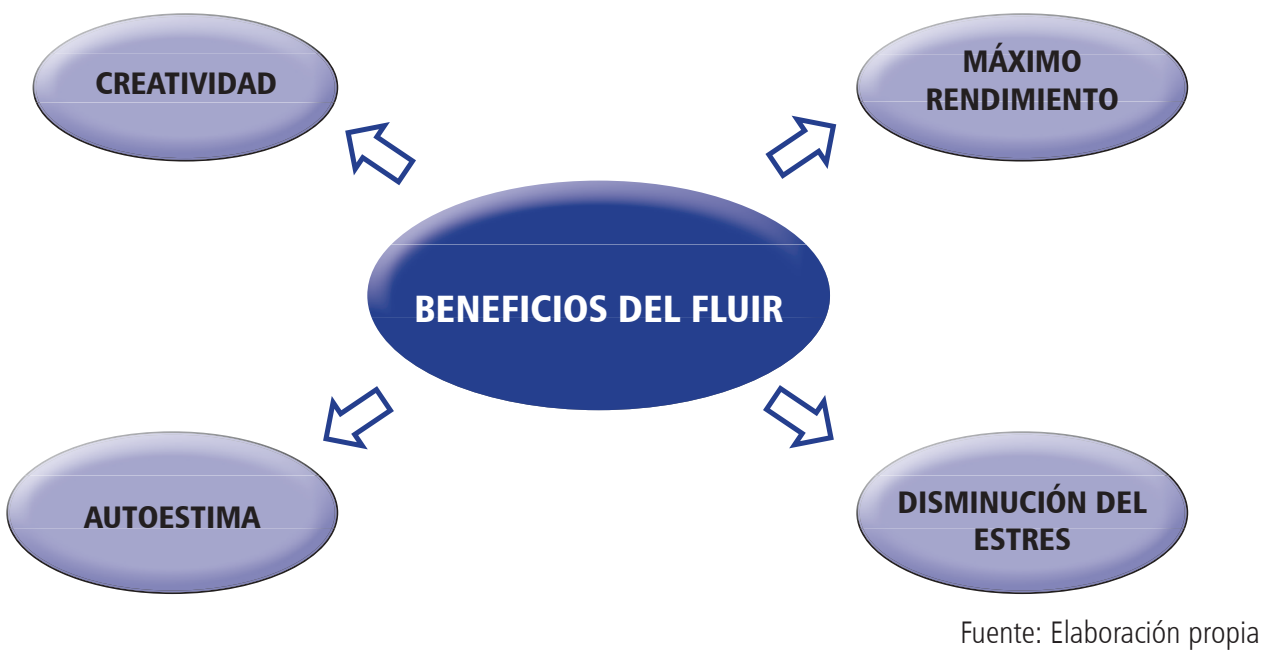

Una de las áreas en las que la experiencia de fluidez parece que puede ser potencialmente útil es en la educación. Se ha demostrado que el grado en que los estudiantes disfrutan de un curso determinado predice mejor los resultados finales que las medidas previas de logro escolar y aptitud. Para facilitar la aplicación de la experiencia de fluidez en la educación, se han de descubrir y proponer que tipos de actividades disfrutan más los estudiantes; por ejemplo que los docentes utilicen más técnicas narrativas-ilustrativas en sus clases, acceso y utilización real por parte del estudiantado de las nuevas tecnologías, proyectos de investigación acción en distintas áreas, utilización de juegos cooperativos didácticos en un clima de diálogo permanente; en fin ubicar situaciones o contextos de acción-concentración y reto que movilicen a los docentes y a los estudiantes. Se aspira que los profesores reelaboren sus estrategias de aprendizaje para que sean retadoras y gratificantes al estudiantado, de manera que este colectivo tenga la oportunidad de llegar a la experiencia de fluidez. Asimismo, podrán encontrar un mayor sentido a las actividades propuestas para el aprendizaje de nuevos contenidos, que se pueden traducir en una mayor motivación hacia las tareas escolares. Realmente es mucho lo que podría hacerse para mejorar el proceso educativo aumentando el disfrute que potencialmente ofrece y en general, todos los participantes en las actividades escolares podrán decidir si deberían hacer cambios en sus vidas escolares para obtener una mayor satisfacción y bienestar. 


\section{REFERENCIAS}

Cataluña, D. y Fiz, J. (2014). Psicología Positiva: Pautas para incrementar tu felicidad. España: Ediciones Dauro.

Cabaco, A.S. (2009). Memoria reparadora: perspectiva psicológica. Naturaleza y Gracia, 57 (2), p. 341-364.

Cabaco, A.S. (2011). Memoria, identidad y sentido vital. Lección Inaugural en la Solemne Apertura del Curso Académico 2011/2012. Universidad Pontificia de Salamanca. Salamanca: Publicaciones de la UPSA.

Cabaco, A. S. (2012). Psicología Positiva e Inteligencia Emocional en un mundo en cambio. Conferencia en XIX Congreso Internacional de INFAD. Lisboa: Universidade Lusófona de Humanidades e Tecnologías.

Cabaco, A.S., Risco, A. y Salvino, E. (2008). Desarrollo de competencias actitudinales en la educación superior: búsqueda de sentido vital y felicidad. Naturaleza y Gracia, 55 (3), 697-721.

Casallo, M. (2008). Prácticas en psicología positiva. Lugar: Argentina.

Chico, E. L. (2002). Optimismo disposicional como predictor de estrategias de afrontamiento. Psicothema, 14 (3), 544-550.

Contreras, F. y Esguerra, G. (2006). Psicología positiva: una nueva perspectiva en psicología. Colombia: Revista Diversitas.

Csikszetmihalyi, M. (1997). Fluir. Barcelona: Kairós.

Csikszetmihalyi, M. (1998). Creatividad. El Fluir y la Psicología del Descubrimiento y la Invención. Barcelona: Paidós

Cuadra, H. y Florenzano, R. (2003). El bienestar subjetivo: hacia una psicología positiva. Revista de Psicología de la Universidad de Chile, 12 (1), 83-96.

Delors, J. (1996). La educación encierra un tesoro. Informe a la UNESCO de la Comisión internacional sobre la educación para el siglo XXI. Madrid: Santillana/UNESCO.

Fernández, A. y Fernández, I. (2009) Crítica y alternativas a la significación estadística en el contraste de hipótesis. Madrid: Muralla.

Fernández-Abascal, E.G. (2008). Las emociones positivas. Madrid: Pirámide.

Gancedo, M. (2008). Historia de la psicología positiva. Antecedentes, aportes y proyecciones. En M.M. Casullo (Ed.), Prácticas en psicología positiva. Buenos Aires: Lugar. 
Gancedo, M. (2009). Psicología Positiva: posible futuro y derivaciones clínicas. Psicodebate, 9, $15-26$.

Godoy, J.F. (1999). Psicología de la Salud: delimitación conceptual. En M.A. Simón (Ed.), Manual de Psicología de la Salud: Fundamentos, Metodología y Aplicaciones (pp. 39-65). Madrid: Biblioteca Nueva.

Haidt, J. (2006). The Happiness Hypothesis. New York: Basic Books.

Lupano, M. L y Castro Solano (2010). Psicología Positiva: Análisis de su surgimiento. Ciencias Psicológicas. Vol.4 Nro. 1.

Ortiz, J., Ramos, N., y Vera-Villarroel, P. (2003). Optimismo y salud: estado actual e implicaciones para la Psicología Clínica y de la Salud. Suma Psicológica. 10(1), 119-134.

Peterson, C., Maier, S.F. \& Seligman, M.E.P. (1993). Learned Helplessness. A theory for the age of personal control. New York: Oxford University Press.

Peterson, C. y Seligman, M.E.P. (Eds.) (2004). Character strengths and virtues: a handbook and classification. Washington, DC: American Psychological Association and Oxford University Press.

Ryan, R.M. y Deci, E.L. (2000). Self-determination theory and the facilitation of intrinsic motivation, social development, and wellbeing. American psychologist, 55, 68-78.

Ryan, R.M. y Lynch, J.H. (1989). Emotional autonomy versus detachment: Revisiting the vicissitudes of adolescence. Child Development, 60, 340-356.

Salvat, Ed. (1976). Diccionario terminológico de ciencias médicas. Undécima edición. Barcelona: Salvat.

Seligman M.E.P. (1998). Learned optimism: How to change your mind and your life. (2a. ed.). New York, NY: Pocket Books.

Seligman, M.E.P. (1999). The president's address. American Psychologist, 54, 559-532.

Seligman, M. y Csikszentmihalyi, M. (2000). Positive Psychology: An introduction. American Psychologist, 55 (1), 5-14.

Seligman, M.E.P. \& Christopher, P. (2000). Positive Clinical Psychology. New York, NY: Pocket Books.

Seligman, M. (2002). La auténtica felicidad. Barcelona: Vergara.

Seligman, M.E.P. (2003). La auténtica felicidad. Barcelona, España: Vergara

Seligman, M. (2003). Positive psychology: Fundamental assumptions. American Psychologist, 126127. 
Seligman M.E.P. y Peterson, C. (2003). Positive clinical psychology. En L.G. Aspinwall \& U.M. Staudinger (Eds.). A Psychology of human strengths: Fundamental questions and future directions for a positive psychology (pp. 305-317). Washington, D.C: American Psychological Association.

Seligman, M.E.P. (2005). La auténtica felicidad (M. Diago y A. Debrito, Trads.). Colombia: Imprelibros, S.A. (Trabajo original publicado en 2002).

Seligman, M. E. P. (2011). Flourish: a visionary new understanding of happiness and well-being. New York: Free Press.

Snyder, C. R. (2002). Hope theory: Rainbows of the mind. Psychological Inquiry, 13, 249-275.

Vázquez, C. (2006). La Psicología Positiva en perspectiva. En Papeles del Psicólogo, 27, 1-2.

Vázquez, C. y Hervás, G. (2008). Psicología Positiva Aplicada. Bilbao: Desclée de Brouwer.

Vázquez y Hervás. A., C. (2007). Psicología positiva: La ciencia de la felicidad. Paidós.

Vázquez, C., Sánchez, A. y Hervás, C. (2008). Intervenciones psicológicas para la promoción del bienestar. En C. Vázquez y G. Hervás (Eds.), Psicología positiva aplicada (pp. 41-71). Bilbao: Desclee de Brower.

Vázquez, C. y Hervás, G. (2009). La ciencia del bienestar. Fundamentos de una psicología positiva. Madrid: Alianza Editorial.

Sheldon, K.M. \& King, L. (2001). Why positive psychology is necessary. American Psychologist, 56, 216-217.

Valliant, G. (2002). Aging well. Boston: Little Brown and Company.

Vázquez, C. (2006). La psicología positiva en perspectiva. Papeles del Psicólogo, 27(1), 1-2.

Vázquez, Carmelo y Hervás, Gonzalo (ed.) (2008). Psicología Positiva Aplicada. Ed. Desclee de Brower.

Vázquez, Carmelo y Hervás, Gonzalo (ed.) (2009). La ciencia del bienestar: Fundamentos para una psicología positiva. Ed. Alianza.

Vera, B. (2008). Psicología positiva. Una nueva forma de entender la psicología. Papeles del psicólogo, 27 (1), 3-8. 